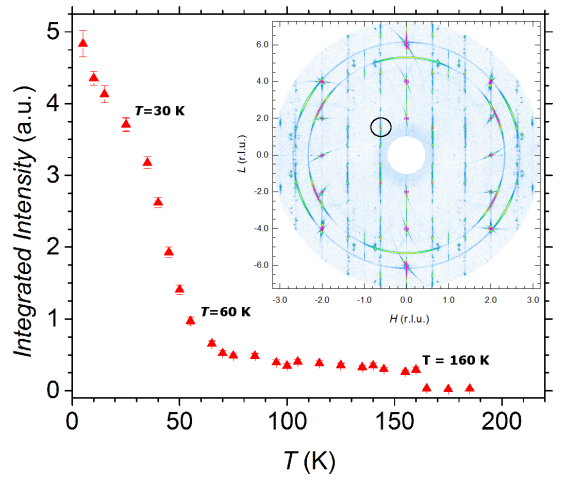

Figure 1. Temperature-dependent scan along $(-0.62, \mathrm{k}, 1.46)$ on $\mathrm{Pr}_{2} \mathrm{NiO}$ single crystal, showing a three-step transition. The scanned magnetic peak is marked in the $(\mathrm{HOL})$ reciprocal map, where a combined 2D and 3D magnetic order is $\operatorname{seen}^{[2]}$.

Keywords: Ruddlesden-Popper, Neutron Scattering, Magnetism, Oxygen Superstructure, Fuel Cell, Oxygen Transport

\section{MS24-P3 Electron density analysis in Quantum Magnets}

Rebecca Scatena ${ }^{1}$, Leonardo H.R. Dos Santos ${ }^{1}$, Arianna Lanza ${ }^{1,2}$, Nicola Casati ${ }^{2}$, Björn Wehinger ${ }^{3}$, Christian Rüegg ${ }^{3}$, Mariusz Kubus $^{1}$, Karl Krämer ${ }^{1}$, Lukas Keller ${ }^{3}$, Tom Fennell ${ }^{3}$, Alun Biffin ${ }^{3}$, Piero Macchi ${ }^{1}$

1. Department of Chemistry and Biochemistry, University of Bern, Freiestrasse 3, 3012, Bern, Switzerland

2. Laboratory for Synchrotron Radiation, Paul Scherrer Institute, 5232 Villigen - PSI Switzerland

3. Laboratory for Neutron Scattering and Imaging, Paul Scherrer Institute, 5232 Villigen - PSI Switzerland

email: rebecca.scatena@dcb.unibe.ch

We have investigated the correlation between the accurate electron density distribution and the magnetic properties of two metal-organic polymeric quantum magnets, the $\left\{\left[\mathrm{Cu}(\mathrm{pyz})_{2} \mathrm{Cl}_{3} \mathrm{BF}_{4}\right\}_{n}\right.$ and the $\left\{\left[\mathrm{Cu}(\mathrm{pyz}){ }_{2} \mathrm{Br}\right] \mathrm{BF}_{4}\right\} \quad$ (pyz $={ }^{2}$ pyrazine), using high resolution ${ }^{2}$ single ${ }^{n}$ crystal $\mathrm{X}$-ray diffraction and density functional theory (DFT) calculations in the crystalline state as well as in the gas phase on selected fragments of the framework. Topological Analysis based on Quantum Theory of Atoms in Molecules (QTAIM) has been applied to characterize the possible magnetic exchange coupling constants. Electron density analysis confirmed the orientation of the magnetic orbital. The magnetic properties have been examined and correlated with the topological and integrated properties of the electronic distribution. This has enabled the detailed rationalization of the experimental antiferromagnetic exchange coupling constants in terms of the interchain $\mathrm{Cu}-\mathrm{Cu}$ interactions. Molecular orbital and spin density analysis have been used to identify the atomic and group sources of magnetism. In particular, halogens and pyrazine act as typical non-innocent ligands, with large part of the spin density developed on their atoms. In both cases, the experimentally observed antiferromagnetic coupling can be explained by the copper-copper super-exchange coupling mediated by the pyrazine ligands. Moreover, our results suggest a non-negligible coupling through the halogens although the electron density shows a much weaker interaction than the one observed along pyrazine. From this study, it is clear that systematic electron density analysis on transition metal compounds could lead to a better understanding of the super exchange mechanism with a topological description of the involved interactions.

Keywords: MOF quantum-magnets elecron-density 